\title{
Escolha da via cirúrgica para tratamento das fraturas cervicais
}

The choice of surgical approach for treatment of cervical fractures

Elección de la vía quirúrgica para el tratamiento de las fracturas cervicales

\author{
Olavo Biraghi Letaif' \\ Marcelo Loquette Damasceno' \\ Alexandre Fogaça Cristante ${ }^{2}$ \\ Raphael Martus Marcon ${ }^{2}$ \\ Alexandre Sadao lutaka ${ }^{2}$ \\ Reginaldo Perilo Oliveira ${ }^{3}$ \\ Tarcísio Eloy Pessoa de Barros Filho ${ }^{4}$
}

\section{RESUMO}

Objetivo: definir características epidemiológicas da população vitima$\mathrm{da}$, classificar as fraturas subaxiais e analisar como foram os tratamentos cirúrgicos, tendo como desfecho a via cirúrgica escolhida - anterior, posterior ou combinada - reunindo tais dados para observar padrões de tratamento para o melhor cuidado desses doentes. Métodos: análise retrospectiva em prontuários médicos de 222 pacientes atendidos e tratados entre o ano de 2004 e o mês de Março de 2009 com fraturas, fraturas-luxações e luxações cervicais. Desses 222 pacientes, 163 correspondiam àqueles que tinham fraturas subaxiais classificáveis pelo método $\mathrm{AO}$, ou seja, correspondiam a aproximadamente $73,4 \%$ do total. Resultados: dentre os pacientes, $83 \%$ eram homens e aproximadamente $78 \%$ tinham entre 21 e 60 anos. Foram classificados como Tipo A 54 pacientes, e 50\% foram operados $-85,18 \%$ via anterior, com corpectomia associada ou não à artrodese; foram classificados como

\section{ABSTRACT}

Objective: to define the epidemiologic characteristics of the involved patients, to classify the subaxial fractures to study how the patients have been surgically treated, with the chosen surgical approach (anterior, posterior or combined) being the main goal of the study, gathering these data in order to observe patterns of treatment for better care of the patient. Methods: the medical history of 222 patients with cervical fractures, fracture-dislocations and dislocations, between the year of 2004 and March 2009, was retrospectively analyzed. Among these 222 patients, 163 of them had subaxial fractures that were eligible to the AO Classification system, corresponding to $73.4 \%$ of the total. Results: $83 \%$ of the patients were male and approximately $78 \%$ were aged between 21 and 60. Fiftyfour patients were classified as Type $A$, and $50 \%$ had surgery $-85,18 \%$ by anterior approach, with corpectomy associated or not with arthrodesis; 77 patients were classified as Type

\section{RESUMEN}

Objetivo: definir las características epidemiológicas de la población víctima, clasificar las fracturas subaxiales, y analizar cómo los tratamientos quirúrgicos fueron realizados, teniendo ellos mismos como resultado la manera quirúrgica elegida - anterior, posterior o combinada -, y juntar estos datos para observar estándares del tratamiento para el cuidado óptimo de esta gente enferma. Métodos: análisis retrospectiva en prontuarios médicos de 222 pacientes atendidos y tratados, entre 2004 y Marzo de 2009 con fracturas, fracturas-luxaciones y luxaciones cervicales. De estos 222 pacientes, 163 correspondieron a los que tenían fracturas subaxiales clasificables por el método $A O$, es decir, aproximadamente un $73.4 \%$ del total. Resultados: los $83 \%$ de los pacientes eran hombres y el aproximadamente $78 \%$ tenian entre 21 y 60 años de la edad. Habian sido clasificados como Tipo A, 54 pacientes, el 50\% habian sido operados - el $85.18 \%$, vía anterior, con corpectomía asociada o no

\footnotetext{
Instituto de Ortopedia e Traumatologia do Hospital das Clínicas, Faculdade de Medicina, Universidade de São Paulo - USP -São Paulo (SP), Brasil.

'Médico Residente do Grupo de Coluna do Instituto de Ortopedia e Traumatologia do Hospital das Clínicas da Faculdade de Medicina da Universidade de São Paulo - USP - São Paulo (SP), Brasil.

${ }^{2}$ Médico Assistente do Grupo de Coluna do Instituto de Ortopedia e Traumatologia do Hospital das Clínicas da Faculdade de Medicina da Universidade de São Paulo - USP - São Paulo (SP), Brasil.

${ }^{3}$ Médico Chefe do Grupo de Coluna do Instituto de Ortopedia e Traumatologia do Hospital das Clínicas da Faculdade de Medicina da Universidade de São Paulo - USP - São Paulo (SP), Brasil.

4Professor Titular do Instituto de Ortopedia e Traumatologia do Hospital das Clínicas da Faculdade de Medicina da Universidade de São Paulo - USP - São Paulo (SP), Brasil 
Tipo B 77 pacientes, e $85,7 \%$ foram operados $-77,3 \%$ via posterior, considerando-se a lesão ligamentar; como Tipo C foram classificados 21 pacientes, e $81 \%$ foram operados $94,1 \%$ via posterior; como Múltiplos Níveis foram considerados 11 pacientes, e $54,5 \%$ foram operados $-83,3 \%$ via posterior, nenhum por via anterior isoladamente. Conclusão: os dados obtidos podem contribuir para a padronização do atendimento ao paciente com traumatismo cervical e tornar os resultados do tratamento mais previsíveis. A experiência acumulada e revertida em números facilitará a escolha da via cirúrgica.
$B$, and $85,7 \%$ had surgery - 77,3\% by posterior approach, considering the ligament lesion; 21 patients were classified as Type C, and $81 \%$ had surgery - 94,1\% posterior approach; finally, 11 patients were classified as Multiple Level, and $54,5 \%$ had surgery - $83,3 \%$ by posterior approach, none by anterior approach alone. Conclusion: the data obtained may contribute to the standardization of the care given to the cervical traumatized patient and make the treatment results more predictable. The experience gathered and converted to numbers will help in the choice of the surgical approach.

a la artrodese; fueron clasificados como Tipo B, 77 pacientes, y el $85.7 \%$ que habían sido operados - el $77.3 \%$ vía posterior, teniendo en cuenta el lesión ligamentar; como Tipo C, 21 pacientes fueron clasificados, y el $81 \%$ que habían sido operados - el 94.1\% de la vía posterior; y como Niveles Múltiples, 11 pacientes fueron considerados, $54.5 \%$ fueron operados - el $83.3 \%$ de la vía posterior, ningún por la vía anterior sola. Conclusión: los datos obtenidos pueden contribuir con la normalización del cuidado del paciente con traumatismo cervical y tornar los resultados más predecibles. La experiencia acumulada y invertida en números facilitará la elección de los medios quirúrgicos.

KEYWORDS: Spinal fracture/ epidemiology; Spinal fracture/ classification; Dislocations; Surgical procedures, operative/methods
DESCRIPTORES: Fractura

de la columna cervical/ epidemiología; Fracturas de la columna cervical/ clasificación; Luxaciones; Procedimientos quirúrgicos operativos/métodos

\section{INTRODUÇÃO}

As fraturas cervicais subaxiais têm extrema relevância em nosso meio em virtude de sua alta prevalência, de suas implicações tanto médico-científicas quanto socioeconômicas $^{1-3} \mathrm{e}$ em relação ao perfil dos pacientes vitimados com esses traumas ${ }^{4-12}$.

Este trabalho teve como objetivo definir características epidemiológicas da população vitimada, classificar as fraturas de acordo com a classificação $\mathrm{AO}$ para fraturas cervicais subaxiais ${ }^{13}$, indicar o tratamento cirúrgico a ser realizado, a via cirúrgica a ser escolhida e quais as complicações de cada uma delas ${ }^{14}$.

Este estudo não se propôs a determinar conduta ou protocolo de tratamento ideal, mas sim reunir os dados colhidos, observar padrões de tratamento empregados nos tipos específicos de fratura e interpretar os resultados para que isso se reverta, futuramente, no melhor cuidado desses pacientes.

\section{MÉTODOS}

Avaliação retrospectiva de prontuários de 222 pacientes atendidos e tratados no Instituto de Ortopedia e Traumatologia (IOT) do Hospital das Clínicas da Faculdade de Medicina da Universidade de São Paulo (USP) nos anos de 2004 (36 pacientes), 2005 (47 pacientes), 2006 (28 pa- cientes), 2007 (53 pacientes), 2008 (42 pacientes) e 2009 até Março (16 pacientes) com fraturas, fraturas-luxações e luxações cervicais.

Desses 222 pacientes, 163 correspondiam àqueles que tinham fraturas subaxiais classificáveis pelo método $\mathrm{AO}^{13}$, ou seja, aproximadamente $73,4 \%$ do total. Os outros 59 pacientes apresentavam fraturas de $\mathrm{C} 1, \mathrm{C} 2$ ou subaxiais de elementos posteriores isoladamente, sem fratura do corpo vertebral, lesão ligamentar ou rotacional, não sendo, portanto, contabilizados para efeitos deste estudo.

A classificação da AO foi escolhida por ser amplamente utilizada, ter grande reprodutibilidade científica, ter alto grau de concordância interobservador e apresentar boa correlação entre grau de energia/gravidade com prognóstico clínico. Nessa classificação são utilizadas identificações alfanuméricas nas quais 5 corresponde à região da coluna, 1 corresponde à cervical e, na sequência, as letras $\mathrm{A}, \mathrm{B}$ e $\mathrm{C}$ correspondem ao mecanismo de trauma e às características da fratura. Neste estudo, as subdivisões de A, B e C não foram utilizadas com o intuito de simplificação - A: corresponde às fraturas do corpo cervical causadas por mecanismo de compressão axial; B: corresponde às lesões cervicais com mecanismo de distração envolvido (flexão ou extensão); C: corresponde às lesões cervicais em que houve mecanismo rotacional de trauma. 
É possível traduzir as lesões cervicais do tipo A como fraturas e as lesões dos tipos $\mathrm{B}$ e C como fraturas-luxações ou luxações. Essa consideração se faz pertinente no que tange, quando oportuno, ao emprego do halo craniano com o objetivo de redução e não somente de estabilização. Foram considerados à parte os pacientes que apresentaram múltiplos níveis de lesão, ou seja, duas ou mais lesões com classificação AO semelhante ou diferente em níveis cervicais distintos, contíguos ou não.

É importante salientar que muitos desses pacientes receberam como tratamento inicial no nosso serviço o halo craniano (Figuras 1 e 2). A colocação do halo no IOT do Hospital das Clínicas da Faculdade de Medicina da USP é feita no Centro Cirúrgico, em condições estéreis, seguindo os parâmetros clássicos de posicionamento (pinos anteriores posicionados $1 \mathrm{~cm}$ acima das sobrancelhas, na transição do $1 / 3$ médio para a lateral das mesmas; os pinos posteriores, $1-2 \mathrm{~cm}$ acima das orelhas, escolhendo-se o halo mais simétrico possível em relação ao maior diâmetro encefálico, mantendose o bom paralelismo do mesmo). Em nosso serviço, o halo craniano é empregado com dois objetivos: estabilização e redução. Na estabilização, o peso do conjunto é de $5 \mathrm{~kg}$. Quando utilizado para a redução, inicia-se com $5 \mathrm{~kg}$, com acréscimo de 2,3 kg por nível cervical, aumentando-se $1 \mathrm{~kg}$ a cada 30 minutos. Ao longo da redução são monitorados os parâmetros vitais, como frequência cardíaca e respiratória, pressão arterial, nível de consciência e presença de nistagmo.

Neste estudo, considerou-se sucesso de redução quando o exame radiográfico de controle em perfil mostrou boa congruência facetária, bom alinhamento dos elementos vertebrais e ausência de sinais de distração excessiva entre os corpos vertebrais.

Os halos empregados no IOT do Hospital das Clínicas da Faculdade de Medicina da USP são de fabricação da própria Oficina Ortopédica do Instituto.

\section{RESULTADOS}

No estudo foram incluídos 163 pacientes, sendo 135 (83\%) do sexo masculino e 28 (17\%) do sexo feminino.

A distribuição por faixa etária foi:

$-<10$ anos -1 paciente ou $0,6 \%$

- 11-20 anos - 22 pacientes ou $13,5 \%$

- 21-40 anos - 74 pacientes ou $45,4 \%$

- 41-60 anos - 54 pacientes ou $33 \%$

- >60 anos - 12 pacientes ou 7,5\%

Foram classificados como Tipo A 54 pacientes, ou seja, cerca de $33 \%$ do total. Destes, 27 pacientes $(50 \%)$ foram operados conforme a via cirúrgica demonstrada na Tabela 1.

Foram classificados como Tipo B 77 pacientes, ou seja, cerca de $47 \%$ do total. Com relação à cirurgia, 66 pacientes $(85,7 \%)$ foram operados, e a distribuição, segundo a via cirúrgica escolhida, está representada na Tabela 2.

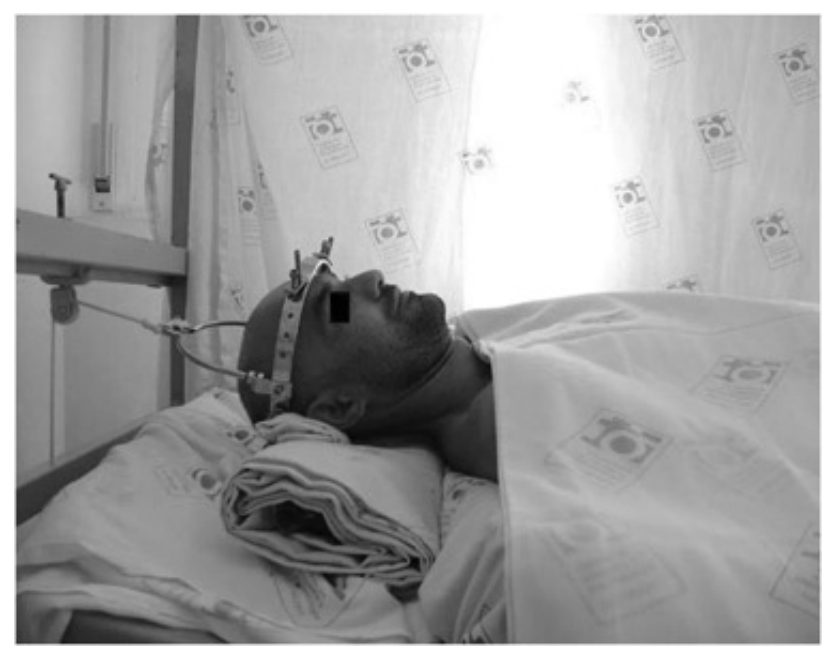

Figura 1

Paciente utilizando halo craniano.

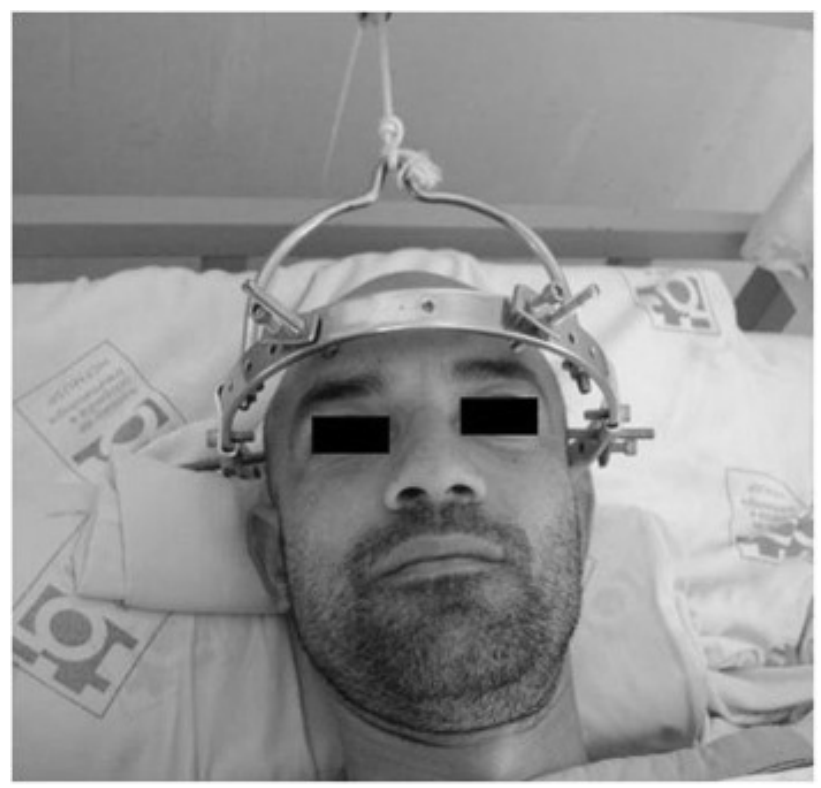

Figura 2

Posicionamento do halo craniano.

TABELA 1 - Resultados do Tipo A

\begin{tabular}{lcc}
\hline Fraturas Tipo A & Pacientes & $\%$ \\
\hline Via anterior & 23 & 85,18 \\
Via posterior & 4 & 14,82 \\
Via combinada & 0 & 0 \\
\hline
\end{tabular}

TABELA 2 - Resultados do Tipo B

\begin{tabular}{lcc}
\hline Fraturas Tipo B & Pacientes & $\%$ \\
\hline Via anterior & 15 & 22,7 \\
Via posterior & 51 & 77,3 \\
Via combinada & 0 & 0 \\
\hline
\end{tabular}


Foram classificados como Tipo C 21 pacientes, ou seja, cerca de $13 \%$ do total, dos quais 17 pacientes $(81 \%)$ foram operados. A distribuição, segundo a via cirúrgica escolhida, encontra-se na Tabela 3.

Foram classificados como Múltiplos Níveis 11 pacientes, cerca de $7 \%$ do total, sendo que 6 pacientes $(54,5 \%)$ foram operados segundo as vias cirúrgicas conforme demonstrado na Tabela 4.

\section{DISCUSSÃO}

A maioria dos pacientes da nossa casuística é do sexo masculino, e $80 \%$ deles se encontram na faixa etária dos 20 aos 60 anos, ou seja, o grupo mais ativo do ponto de vista econômico dentro da sociedade $e^{-6,6-8-12}$.

O tipo de fratura mais prevalente foi do Tipo B, seguido pelo Tipo A. Talvez possamos atribuir esse achado ao fato de que o mecanismo de lesões envolvendo algum grau de distração (acidentes automobilísticos, traumas esportivos, agressões físicas etc.) é mais frequente do que traumas puramente axiais ${ }^{4-6,8-12}$.

É importante salientar que a via cirúrgica escolhida, em princípio, foi uma decisão adotada conforme a experiência do nosso serviço.

Nas lesões do Tipo A, metade desses pacientes foi operada e a maioria $(85 \%)$ pela via anterior, ou seja, a escolha da abordagem cirúrgica foi condizente com o local anatômico da lesão, no caso, o corpo vertebral. Não havendo lesão ligamentar, a via posterior não foi empregada. A maioria desses pacientes foi submetida à corpectomia do nível correspondente, associada ou não à artrodese, com o intuito de dar suporte anterior à coluna cervical fraturada ${ }^{14}$.

Já nas lesões do Tipo B, a quase totalidade desses pacientes foi operada ( $85 \%$ ) e, quando isso ocorreu, diferentemente do Tipo A, realizou-se predominantemente a cirurgia por via posterior, considerando-se a lesão ligamentar posterior presente. Deve-se considerar também a maior frequência do mecanismo em flexão em relação ao de extensão. Os pacientes operados por via anterior tinham lesão ligamentar anterior ou insuficiência de sustentação por fratura associada do corpo vertebral ${ }^{14}$.

Com as lesões do Tipo C, de modo semelhante ao Tipo $\mathrm{B}$, quase todos os casos foram submetidos à cirurgia (81\%), a qual foi realizada quase exclusivamente por via posterior (95\%). Isso pode ser explicado, pois, nesses casos de lesão com componente rotacional cervical, a fratura
TABELA 3 - Resultados do Tipo C

\begin{tabular}{lcc}
\hline Fraturas Tipo C & Pacientes & $\%$ \\
\hline Via anterior & 1 & 5,9 \\
Via posterior & 16 & 94,1 \\
Via combinada & 0 & 0 \\
\hline
\end{tabular}

TABELA 4 - Resultados de Múltiplos Níveis

\begin{tabular}{lcc}
\hline Múltiplos Níveis & Pacientes & $\%$ \\
\hline Via anterior & 0 & 0 \\
Via posterior & 5 & 83,3 \\
Via combinada & 1 & 16,7 \\
\hline
\end{tabular}

do corpo vertebral, quando ocorre, não é tão significativa quanto a lesão ligamentar posterior.

Em relação aos casos com Múltiplos Níveis de lesão, pouco mais da metade dos pacientes foi submetida à cirurgia. Curiosamente, nenhum foi operado por via anterior isoladamente, pois, além da presença de lesão dos elementos posteriores, tecnicamente uma artrodese anterior longa é mais difícil, e a chance da falta de consolidação da artrodese aumenta. Deve-se levar em conta que as lesões nem sempre ocorrem em níveis contíguos, tornando sua abordagem mais complexa. Outro dado digno de nota é que, em todos os casos estudados, apenas uma vez foi empregada a via combinada, justamente em um caso de Múltiplos Níveis, reforçando a necessidade de abordagens bem planejadas e específicas para essas situações complicadas.

\section{CONCLUSÃO}

De certo modo, é melhor compreender as causas desses traumas cervicais e as características dessa população em risco, adotando-se medidas preventivas de educação, orientação e conscientização pública, pois, apesar dos grandes avanços técnicos e tecnológicos nessa área, o tratamento desses pacientes ainda constitui fonte de debates, controvérsias, desafios e preocupação para a maioria dos profissionais de saúde.

A análise individual de cada paciente poderá determinar em quais casos o halo craniano deverá/poderá ser empregado e qual a taxa de sucesso que se pode esperar com esse tratamento.

\section{REFERÊNCIAS}

1. Herkowitz H, Garfin S, Eismont F, Bell G, Balderston R. RothmanSimeone, The Spine. $5^{\text {th }}$ edition. Philadelphia: Saunders; 2006.
2. Leventhal MR. Fractures, dislocations, and fracture-dislocations of spine. In: Canale TS, editor. Campbell's operative orthopaedics. $10^{\text {th }}$ ed. Philadelphia: Mosby; 2003. p. 1597690.
3. Müller CW, Hüfner T, Gösling T, Krettek C. [Traumatology of the spine]. Chirurg. 2008;79(10):918, 9206. German.

4. Leucht P, Fischer K, Muhr G, Mueller EJ. Epidemiology of traumatic spine fractures. Injury. 2009;40(2):166-72. 
5. Pickett GE, Campos-Benitez M, Keller JL, Duggal N. Epidemiology of traumatic spinal cord injury in Canada. Spine (Phila Pa 1976). 2006;31(7):799-805.

6. Hadała M, Barrios C. Sports injuries in an America's Cup yachting crew: A 4-year epidemiological study covering the 2007 challenge. J Sports Sci. 2009;27(7):711-7.

7. Smith JL, Ackerman LL. Management of cervical spine injuries in young children: lessons learned. J Neurosurg Pediatr. 2009;4(1):64-73.

8. Lenehan B, Boran S, Street J, Higgins T, McCormack D, Poynton AR. Demographics of acute admissions to a National Spinal Injuries Unit. Eur Spine J. 2009;18(7):938-42.
9. Hu J, Yang KH, Chou CC, King AI. A numerical investigation of factors affecting cervical spine injuries during rollover crashes. Spine (Phila Pa 1976). 2008;33(23):2529-35.

10.Wang MC, Pintar F, Yoganandan N, Maiman DJ. The continued burden of spine fractures after motor vehicle crashes. J Neurosurg Spine. 2009;10(2):86-92.

11.Obalum DC, Giwa SO, Adekoya-Cole TO, Enweluzo GO. Profile of spinal injuries in Lagos, Nigeria. Spinal Cord. 2009;47(2):134-7.

12.Boden BP, Jarvis CG. Spinal injuries in sports. Phys Med Rehabil Clin N Am. 2009;20(1):55-68.

13.Aebi M, Arlet V, Webb J. AO Spine Manual - Principles and Techniques, Clinical Applications. Stuttgart: Thieme Verlag; 2007.
14.Lambiris E, Kasimatis GB, Tyllianakis M, Zouboulis P, Panagiotopoulos E. Treatment of unstable lower cervical spine injuries by anterior instrumented fusion alone. J Spinal Disord Tech. 2008;21(7):500-7.

\section{Correspondência}

Olavo Biraghi Letaif

Disciplina de Coluna Vertebral e Trauma Raquimedular, CENATRA, do Instituto de Ortopedia e Traumatologia do Hospital das Clínicas da Faculdade de Medicina da USP

Rua Dr. Ovídio Pires de Campos, 333, $2^{\circ}$ andar - Cerqueira César

CEP 05403-0 10 - São Paulo (SP), Brasil

E-mail: cenatra.ioł@hcnet.usp.br 